\title{
An Incarcerated Divergent and Axial Dislocation of Ankle without any Fracture
}

\author{
Vasantha Kumar Ramsingh \\ Department of Trauma and Orthopaedics, Nevill Hall Hospital, Abergavenny, NP7 7EG, United Kingdom.
}

Corresponding Author:

Dr Vasantha Kumar Ramsingh

Email: drvramsingh@yahoo.co.uk

This is an Open Access article distributed under the terms of the Creative Commons Attribution License (creativecommons.org/ licenses/by/3.0).

Received Accepted

Published

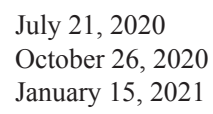

\begin{abstract}
Background: Ankle fracture is a common case encountered in the emergency department. It is not uncommon to come across a rare fracture dislocation patterns on a day-to-day practice. Our aim is to create awareness of a rare type of ankle dislocation without any obvious fracture. Case Report: We report a case of an axial divergent incarcerated dislocation of ankle with no fracture. This was reduced by a gentle repeated dialing movement and subsequently fixed with syndesmosis screws. Conclusion: A tibiotalar dislocation without any fracture is a rare type of ankle injury. This should be treated as any other fracture dislocation of the ankle. Awareness of this type of injury should prompt to get early radiological examination and appropriate subsequent management.
\end{abstract}

Keywords: Ankle Fractures, Bone Screws, Emergency Service, Fracture Dislocation, Joint Dislocations.

\section{Introduction}

Ankle fracture dislocation is a common occurrence in clinical practice. However, pure tibiotalar dislocations with no associated fractures are rare injuries reported in the literature. We report a case of an axial divergent incarcerated dislocation of the ankle with no fractures. Our patient was informed that data concerning the case would be submitted for publication, and he consented.

\section{Case Report}

A 23-year-old male presented to the accident and emergency department 4 hours following the motorbike accident. He fell off a motorbike at $60 \mathrm{MPH}$ speed and sustained left ankle injury. On initial examination the ankle was found to be deformed and externally rotated with very tight skin on the medial side. However there were no open wounds and the vascularity was intact. No initial radiographs were obtained in order to expedite the emergent manipulative reduction. Closed manipulation under conscious sedation with intravenous morphine was attempted by the Accident and Emergency doctors. After two failed attempts at manipulation, the case was referred to the on call orthopaedic surgeon.

The on call orthopaedic surgeon attempted to reduce the ankle under conscious sedation of intravenous morphine and midazolam using longitudinal traction but it was found to be solid and incarcerated. It was thought that a possible fracture fragment was hindering the relocation hence immediate portable radiographs were obtained to study the nature of the injury. Reviewing both the anteroposterior and lateral radiographs revealed an unusual divergent and axial dislocation of the ankle with no associated fractures [Fig.1a,b]. The talus was incarcerated between the distal tibia and fibula with wide diastasis of the syndesmosis. The direction of the talar dislocation was proximal (axial) and posterior. Further clinical and radiological examination excluded fracture at the proximal fibula. After studying the radiographs the ankle was successfully relocated using the 
technique described below without needing formal anaesthetic.

Under the conscious sedation of intravenous morphine and midazolam with the knee in flexion, heel was held with the left hand and the foot was held with the right hand. A gentle dialing movement was created by firstly externally rotating the foot followed by internally rotating. With the repeated dialing movements and sustained longitudinal traction the ankle was clunked back and reduced. Post manipulation anteroposterior and lateral check radiographs were obtained. The lateral view confirmed good reduction [Fig.2a]; however in the anteroposterior film talar shift $(2.5 \mathrm{~mm})$ was noted [Fig.2b]. Decision was made to examine the ankle under anaesthetic and fix accordingly. Examination under anaesthetic revealed unstable syndesmosis with talar shift, however the ankle was completely reducible with no deltoid ligament interposition. No fractures were identified on screening. The syndesmosis was reduced stabilized using two fully threaded $4.5 \mathrm{~mm}$ tricortical screws. The final position was satisfactory and the ankle was stable following fixation [Fig.3a,b]. A below knee cast was applied and the patient was mobilized, nonweight bearing for 8 weeks.

The patient was followed up in the fracture clinic at regular intervals with check radiographs. Patient returned to work at three months. Weight bearing check radiographs taken at four months, confirmed good ankle alignment with no talar shift [Fig.4a,b]. At one year final follow up patient had full range of pain free ankle movements.

\section{Discussion}

The ankle is a hinge joint, it does not have a discrete stabilizing structure anteriorly or posteriorly and is reinforced with strong collateral ligaments. The ligaments are usually stronger than the malleoli, so that ankle dislocation is usually accompanied by fracture [1]. Furthermore the distal tibia and the medial and lateral malleoli form a mortise
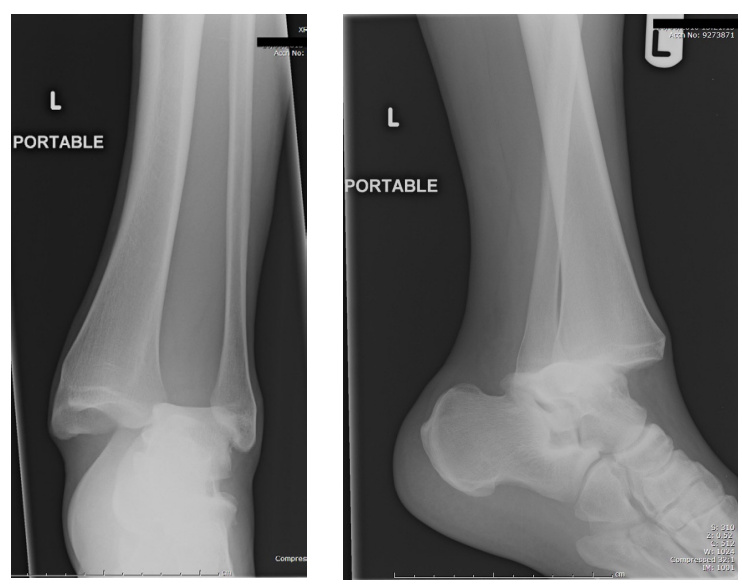

Fig.1: (a) Pre-reduction anteroposterior ankle radiograph showing incarcerated divergent and axial dislocation of ankle; (b) Pre-reduction lateral ankle radiograph showing posterior displacement.
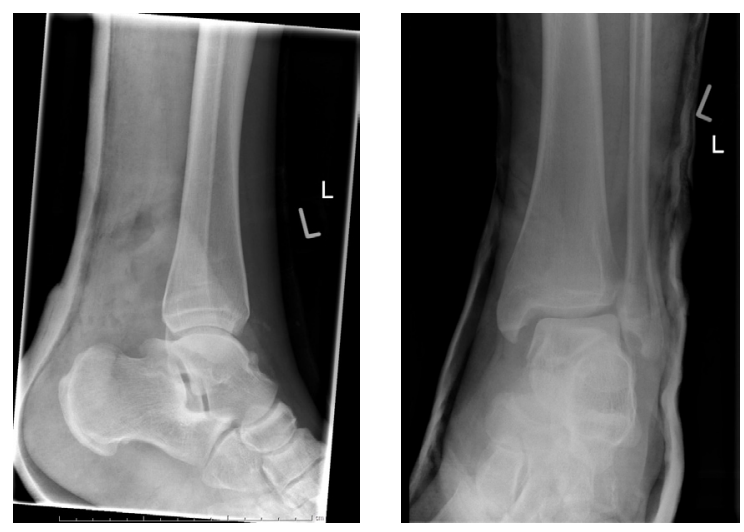

Fig.2: (a) Post-reduction lateral ankle radiograph showing good reduction; (b) Post-reduction anteroposterior ankle radiograph showing reduced ankle with talar shift.
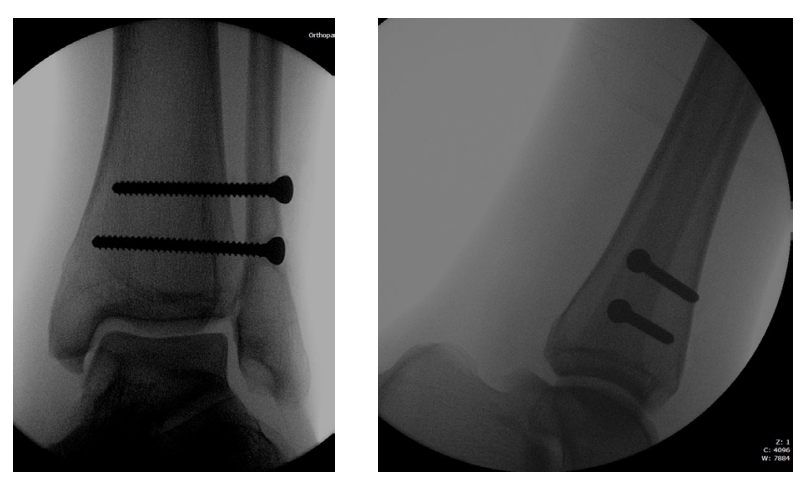

Fig.3: (a) Intra-operative anteroposterior ankle image intensifier picture showing ankle fixation; (b) Intra-operative lateral ankle image intensifier picture. 
into which the talar dome fits with the malleoli limiting rotation of the talus, giving mechanical stability [2]. Therefore pure tibiotalar dislocations without associated malleolar fracture are very rare [3-9]. The common type of ankle dislocation is posteromedial, because of tendency to land in plantar flexion and inversion of the ankle when falling from height [10]. However cases of pure posterior, anterior, anterolateral ankle dislocations have also been described. Upward dislocation of ankle is exceptional; this complex injury has a combination of deltoid ligament rupture with syndesmotic rupture. The three-plane dislocation of the talus includes lateral, posterior and upward (axial). The talus was incarcerated in between tibia and fibula. Although the patient cannot remember the exact mechanism of injury it appears that a combination of axial load and external rotation resulted in this unusual injury. This rare injury does not fit in to any of the current classifications systems of ankle fracture dislocations (LaugeHansen/ Weber).

Manipulative reduction of this dislocation posed a challenge as the talus was locked between the distal tibia and fibula. In general, manipulative reduction without pre-radiographic assessment is an ideal and acceptable practice to reduce the soft tissue complications. Failure at the initial attempts of reduction in this case necessitated obtaining prereduction radiographs. As the routine method of closed reduction failed and there were no fracture fragments blocking the reduction and the medial soft tissues were threatened, this has led to the dilemma of whether to proceed with open reduction or pursue with other alternative closed method. The described new closed reduction method and the subsequent surgical fixation of syndesmosis resulted in a good outcome. Awareness of this rare injury and the described closed reduction method will help in the management of similar cases without need for open reduction.
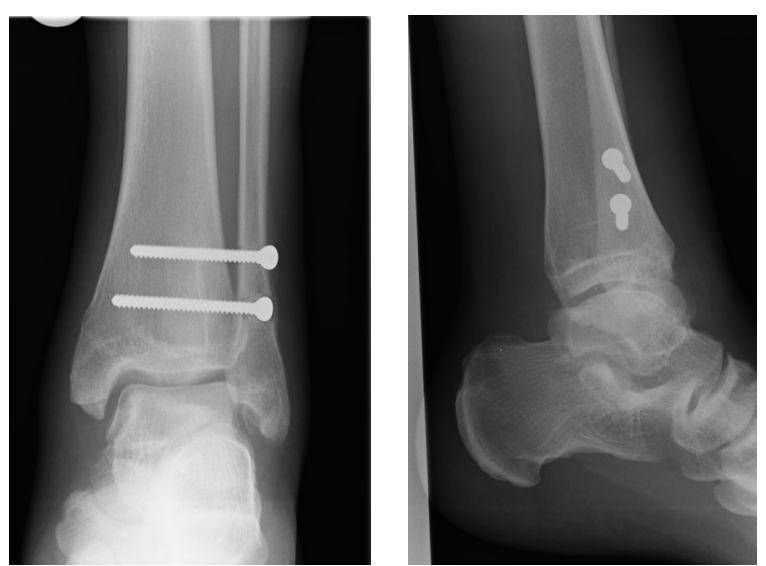

Fig.4: (a) Follow up anteroposterior ankle radiograph showing maintenance of reduction; (b) Follow up lateral ankle radiograph showing maintenance of reduction.

\section{Conclusion}

Tibiotalar dislocation without any fracture is a rare type of ankle injury. This should be treated as any other fracture dislocation of the ankle. Awareness of this type of injury should prompt to get early radiological examination and appropriate subsequent management.

Contributors: VKR did manuscript writing and editing, patient management and will act as study guarantor. The author approved the final version of the manuscript and is responsible for all aspects of the study.

Funding: None; Competing interests: None stated.

\section{References}

1. Wilson MJ, Mlichels AA, Jacobson EW.Ankle dislocation without fracture. J Bone Joint Surg. 1939;21:198-204.

2. Toohey J, Worsing R. A long term of follow up study of tibiotalar dislocations without associated fractures. Clin Orthop. 1989;239:207-210.

3. Elisé S, Maynou C, Mestdagh H Forgeois F, Labourdette P. Simple tibiotalar luxation. Apropos of 16 cases. Acta Orthop Belg. 1998;64:25-34.

4. Rivera F, Bertone C, De Martino M, Pietrobono D. Pure dislocation of the ankle: three case reports and literature review. Clin Orthop Relat Res. 2001;382:179-184.

5. Hatori M, Kotajima S, Smith RA, Kokubun S. Ankle dislocation without accompanying malleolar fracture. A case report. Ups J Med Sci. 2006;111:263-268.

6. Wilson AB, Toriello EA. Lateral rotatory dislocation of the ankle without fracture. J Orthop Trauma. 1991;5:9395. 
7. Colville MR, Colville JM, Manoli A 2nd. Posteromedial dislocation of the ankle without fracture. J Bone Joint Surg Am. 1987;69:706-711.

8. Wroble RR, Nepola JV, Malvitz TA. Ankle dislocation without fracture. Foot Ankle. 1988;9:64-74.
9. Agrawal AC, Raza HK, Haq RU. Closed posterior dislocation of the ankle without fracture. Indian J Orthop. 2008;42:360-362.

10. Conwell HE, Key FC. Management of fractures, dislocations and sprains. $7^{\text {th }}$ ed. CV Mosby, St Louis, 1961, pp. 1057-1064. 